\title{
Real rate swaption and zero coupon inflation index swaption
}

\section{Christian Kamtchueng}

\author{
CTK Corp., \\ London, UK \\ and \\ ESSEC, \\ Paris, France \\ Email: christian.kamtchueng@gmail.com
}

\begin{abstract}
Inflation products have been developed in the mid ' 90 s. Over the past decade, the market has increased in size and volume. After the credit crunch and the fear of recession, investors have been very pushy for inflation linked derivatives, the major buyer being pension funds. Some interests have been expressed for real rate pure exposure. We will describe a methodology for the pricing of real rate swaption under LMM SABR.
\end{abstract}

Keywords: inflation; derivatives; real rate; inflation swaption.

Reference to this paper should be made as follows: Kamtchueng, C. (2017) 'Real rate swaption and zero coupon inflation index swaption', Int. J. Bonds and Derivatives, Vol. 3, No. 1, pp.21-43.

Biographical notes: Christian Kamtchueng is the Head Advisory of CTK Corp. He is a Lecturer at ESSEC for the Master 'MSTF'. His research interests include issues related to conceptual pricing theory post Lehman default and derivatives pricing and computation technique in finance. He is the author of research studies published at national and international journals, conference proceedings as well as book such as Introduction to the Fear Pricing Theory and Fear Pricing Theory: Credit and Liquidity Adjustments.

\section{Introduction}

Inflation products have been developed in the mid ' 90 s. Over the past decade, the market has increased in size and volume. After the credit crunch and the fear of recession, investors have been very pushy for inflation linked derivatives, the major buyer being the pension funds. Some interests have been expressed for real rate pure exposure. The inflation market is based on two major liquid instruments the Year on Year and the Zero Coupon swap. The last one providing the compounding $\mathrm{ZC}$ rate against a fixed payment at maturity whereas the YoY swap is based on a exchange between a fixed leg and floating payment composed by the just-set annual inflation rate. The option market on the two products gives views on different volatility regime; the YoY on the forward volatility and the ZC on the CPI variance over the maturity of the swap. The consistencies between the two markets have been a huge quantitative challenge. If most of the literature focus on the index CPI modelling (see, for instance, Mercurio and Moreni, 2006; Wimmer, 
2006; Hinnerich, 2006), Kenyon (2006), Belgrade et al. (2004) and Mercurio and Moreni (2010) modelled the index ratio. We will describe a methodology for the pricing of real rate swaption under LMM SABR (Mercurio and Morinni, 2010).

\section{Notations}

The inflation market is defined first of all by different index definition. We consider a inflation index, by $I(t)$ we denote the value of the index at time $t$.

- $\quad I(t)$, value of the index at time $t$.

- $\quad I_{i}^{f}(t), T_{i}$-forward inflation index at time $t . I_{i}^{f}(t):=E_{i}^{T_{i}}[I(t)]$

- $\quad Z C I I^{I}\left(t, K, T_{n}\right)$, the value of the zero coupon inflation indexed swap at time $t$. At time $T_{n}$, the seller inflation gives $N \times\left[\frac{I\left(T_{n}\right)}{I(0)}-1\right]$ to the buyer who gives in exchange

$N \times\left[(1+K)^{n}-1\right]$. Where $K$ is the contract fixed rate and $N$ the contract nominal. $K$ has been determined to cancel the inflation leg at time

$$
0\left(\left[(1+K)^{n}-1\right]=E^{T_{n}}\left[\frac{I\left(T_{n}\right)}{I(0)}-1\right]\right) \text {. }
$$

- $\quad Y_{Y I I}^{I}\left(t, K,\left(T_{i}\right)_{0 \leq i \leq n}\right)$ the value of the year-on-year inflation-indexed swap at time $t$. At each fixings date $T_{i}$, the seller inflation gives to the buyer $N \tau_{i}\left[\frac{I\left(T_{i}\right)}{I\left(T_{i-1}\right)}-1\right]$.

- IICap - IIFloor $\left(t, k,\left(T_{i}\right)_{0 \leq i \leq n}\right)$ the value of inflation indexed cap and floor at time $t$. At each fixings date $T_{i}$, the seller inflation gives to the buyer $N \tau_{i}\left[w\left(\frac{I\left(T_{i}\right)}{I\left(T_{i-1}\right)}-1-k\right)^{+}\right](w=1$ for a Caplet and -1 for a Flooret $)$.

- ZCIIOption $\left(t, k, T_{n}\right)$ the value of inflation indexed zero coupon swaption at time $t$. At time $T_{n}$, the seller inflation gives to the buyer $N\left[w\left(\frac{I\left(T_{n}\right)}{I(0)}-1-k\right)^{+}\right]$ ( $w=1$ for a payer inflation and -1 for a receiver).

- $\quad L P I\left(t, C, F, T_{n}\right)$ the value of a limited price inflation swap at time $t$. There are many types of LPI, the most common is the following payout:

$$
\begin{aligned}
& N \prod_{i=1}^{n} \tau_{i} \operatorname{Min}\left(\operatorname{Max}\left(\frac{I\left(T_{i}\right)}{I\left(T_{i-1}\right)}, 1+F\right) 1+C\right) \text { which can be rewrite as } \\
& N \prod_{i=1}^{n} \tau_{i}\left[\frac{I\left(T_{i}\right)}{I\left(T_{i-1}\right)}+\left(F+1-\frac{I\left(T_{i}\right)}{I\left(T_{i-1}\right)}\right)^{+}-\left(\frac{I\left(T_{i}\right)}{I\left(T_{i-1}\right)}-1-C\right)^{+}\right] .
\end{aligned}
$$


- RRSwaption $\left(t, k, T_{i}, T_{n}\right.$, Libor $\left.^{\delta}\right)$ the value of the real rate swaption at time $t$. The underlying at expiry $T_{i}$ is a zero coupon swap. The seller pays the $1+$ Libor $^{\delta}$ compounded until maturity $T_{n}$, and receives the index ratio weighted by a fixed rate $1+k$ compounded. $\frac{I\left(T_{n}\right)}{I\left(T_{i}\right)}(1+k)^{n-i}$.

The option payoff at $T_{n}$ is $N\left[w\left(\frac{I\left(T_{n}\right)}{I\left(T_{i}\right)}(1+k)^{n-i}-\prod_{j=i+1}^{n-j}\left(1+\delta^{j} L_{j}^{\delta}\right)\right)^{+}\right]$. If we ignore the volatility of the floating (Libor leg) it is a inflation indexed zero coupon option at expiry $T_{i}$, the value of the floating leg is one in $T_{i}$. With an adjusted strike ZCIIOption $\left(t, \frac{1}{(1+k)^{n-i}}, T_{n}, \frac{N}{(1+k)^{r}}\right)$. It is a forward starting inflation indexed zero coupon option in the first order. In the second order, it is a forward starting spread option on a forward ratio inflation index and a zero coupon.

$$
\begin{aligned}
N\left[w\left(\frac{I\left(T_{n}\right)}{I\left(T_{i}\right)}(1+k)^{n-i}-\prod_{j=i+1}^{n-i}\left(1+\delta^{j} L_{j}^{\delta}\right)\right)^{+}\right] & =N\left[w\left(\frac{I\left(T_{n}\right)}{I\left(T_{i}\right)}(1+k)^{n-i}-\frac{1}{P^{\delta}\left(T_{i}, T_{n}\right)}\right)^{+}\right] \\
& =N\left[w\left(\frac{I\left(T_{n}\right)}{I\left(T_{i}\right)}(1+k)^{n-i}-B A\left(T_{i}, T_{n}\right)\right)^{+}\right]
\end{aligned}
$$

\section{Pricing model}

\subsection{SABR LMM market model}

The intuition of the Mercurio SABR LMM is the following: the $T_{i}$-forward inflation index is a lognormal process

$$
\begin{aligned}
d I_{i}^{f}(t) & =I_{i}^{f}\left[\sum_{j=\beta(t)}^{i} V^{j}(t) d Z_{t}^{j, i}\right] \\
d V^{j}(t) & =V^{j}(t)\left[v^{j} d W_{t}^{j}\right] \\
\rho_{V, Z}^{i, j} d t & =d W_{t}^{j} d Z_{t}^{j, i}
\end{aligned}
$$

with $W^{j}$ Brownian motion under the $Q_{T_{j}}$ forward measure and $Z_{t}^{j, i}$ Brownian motion under the $Q_{T_{i}}$ forward measure and defined recursively as follow: $Z_{t}^{j, i-1}:=Z_{t}^{j, i}-\rho_{i, j}^{F, Z} \frac{\delta L^{i}(t) \sigma_{t}^{r, i}}{1+\delta L^{i}(t)}$. To determine the price of the year on year option, YYCap and YYFloor, we derive the dynamic of the inflation index ratio as the forward index ratio plus a convexity adjustment. 


$$
\begin{aligned}
R_{i} & =E_{t}^{T_{i}}\left[\frac{I_{i}^{f}\left(T_{i}\right)}{I_{i-1}^{f}\left(T_{i-1}\right)}\right] \\
& =\frac{I_{i}^{f}(t)}{I_{i-1}^{f}(t)} e^{\int_{0}^{T_{i}} D(s) d s} \\
& \approx \frac{I_{i}^{f}(t)}{I_{i-1}^{f}(t)} e^{\int_{0}^{T_{i}} \hat{D}(s) d s} \\
& =Y_{i}(t)
\end{aligned}
$$

Therefore, the price of an year on year is given by the Black and Scholes formula

$$
\begin{aligned}
Y Y \text { Caplet }\left(t, T_{i-1}, T_{i}, K:=1+k, w\right) & =w P\left(t, T_{i}\right)\left[Y_{i}(t) N(w d 1)-K N(w d 2)\right] \\
d 1 & =\frac{\ln \left(\frac{Y_{i}(t)}{K}\right)+\frac{1}{2} \sigma^{2}(K)\left(T_{i}-t\right)}{\sigma(K) \sqrt{T_{i}-t}} \\
d 2 & =\frac{\ln \left(\frac{Y_{i}(t)}{K}\right)-\frac{1}{2} \sigma^{2}(K)\left(T_{i}-t\right)}{\sigma(K) \sqrt{T_{i}-t}}
\end{aligned}
$$

with $\sigma(K)$ the volatility from the Hagan SABR formula. In order to price the zero coupon inflation indexed option, we match the variance of the inflation index over the expiry as function of the year on year $V^{i}$ parameters. We suppose the inflation rate $I(t)$ driven by the following dynamic:

$$
\begin{aligned}
d I(t) & =I(t)\left[V d Z_{t}\right] \\
d I(t) & =V(t)\left[u d W_{t}\right] \\
\rho d t & =d W_{t} d Z_{t}
\end{aligned}
$$

In Merton (1976a), Mercurio described some possible proxies for the inflation index SABR parameters

$$
\begin{aligned}
V(0) & =\sqrt{\sum_{i, j=1}^{N} V^{j}(0) V^{i}(0) \rho_{i, j}^{Z} \frac{T_{i} \wedge T_{j}}{T_{N}}} \\
v & =\sqrt{\sum_{i, j, k, l=1}^{N} \bar{V}^{j}(0) \bar{V}^{i}(0) \bar{V}^{k}(0) \bar{V}^{l}(0) \rho_{i, j}^{Z} \rho_{l, k}^{Z} \frac{T_{i} \wedge T_{j} \wedge T_{l} \wedge T_{k}}{T_{N}} v_{j} v_{k} \rho_{j, k}^{V}} \\
v(t) & =\sqrt{\sum_{i, j, k, l=1}^{N} \bar{V}^{j}(0) \bar{V}^{i}(0) \bar{V}^{k}(0) \bar{V}^{l}(0) \rho_{i, j}^{Z} \rho_{l, k}^{Z} v_{j} v_{k} 1_{\left\{t<T_{i} \wedge T_{j} \wedge T_{l} \wedge T_{k}\right\}} \rho_{j, k}^{V}} \\
\hat{V}^{j}(t) & =\frac{V^{j}(t)}{V(t)} \\
\rho & =\frac{\sqrt{\sum_{i, j, k=1}^{N} \bar{V}^{j}(0) \bar{V}^{i}(0) \bar{V}^{k}(0) \rho_{i, j}^{Z} v_{j} 1_{\left\{t<T_{i} \wedge T_{j} \wedge T_{l} \wedge T_{k}\right\}} \rho_{j, k}^{V}}}{v}
\end{aligned}
$$


Therefore given the approximations chosen, Hagan formula can be used to recover our zero coupon inflation indexed market smile. We have to notice that depending on the liquidity and the choice of calibration; one can decide to fit perfectly each year on year inflation indexed option then calibrate the correlation to retrieve the market price of the zero coupon inflation indexed.

\subsection{Forward zero coupon swaption}

For a forward zero coupon swaption, we have to approximate the forward market volatility. We want to find the SABR parameters of the forward ratio $\frac{I\left(T_{n}\right)}{I\left(T_{i}\right)}$

$$
\begin{aligned}
\mathrm{E}_{t}^{T_{n}}\left[\frac{T\left(T_{n}\right)}{I\left(T_{i}\right)}\right] & =\mathrm{E}_{t}^{T_{n}}\left[\frac{I_{n}^{f}\left(T_{n}\right)}{I_{i}^{f}\left(T_{i}\right)}\right] \\
& =\frac{I_{n}^{f}(t)}{I_{i}^{f}(t)} e^{\int_{t}^{T_{n}} D_{n, i}(t) d t}
\end{aligned}
$$

We defined $R^{n, i}:=\frac{I_{n}^{f}\left(T_{n}\right)}{I_{i}^{f}\left(T_{i}\right)}$. By applying Ito formula we have

$$
\begin{aligned}
d R_{t}^{n, i} & =R_{t}^{n, i}\left[D^{n, i}(t) d t+\sum_{j=i+1}^{n} V^{j}(t) d Z_{t}^{j, n}\right] \\
R_{0}^{n, i} & =\frac{I_{n}^{f}(0)}{I_{i}^{f}(0)}
\end{aligned}
$$

We have $Y^{n, i}$ such that

$$
\begin{aligned}
\frac{d Y_{t}^{n, i}}{Y_{t}^{n, i}} & =\sum_{j=i+1}^{n} V^{j}(t) d Z_{t}^{j, n} \\
Y_{0}^{n, i} & =\frac{I_{n}^{f}(0)}{I_{i}^{f}(0)} e^{\int_{t}^{T_{n}} D_{n, i}(t) d t}
\end{aligned}
$$

We have $\bar{Y}^{n, i}$ such that

$$
\begin{aligned}
\frac{d \bar{Y}_{t}^{n, i}}{\bar{Y}_{t}^{n, i}} & =V^{Y} d Z_{t} \\
\bar{Y}_{0}^{n, i} & =Y_{0}^{n, i} \\
d V^{Y} & =v^{Y} V^{Y} d W t \\
\rho^{Y} d t & =d<W, Z>_{t}
\end{aligned}
$$


Therefore, $\bar{Y}^{n, i}$ follows a SABR dynamic,

$$
\begin{aligned}
V^{Y}(0) & =\sqrt{\sum_{k, j=i+1}^{n} V^{j}(0) V^{k}(0) \rho_{i, j}^{Z} \frac{\left(T_{k} \wedge T_{j}-T_{i}\right)}{T_{n}-T_{i}}} \\
v^{Y} & =\sqrt{\sum_{h, j, k, l=i+1}^{n} \bar{V}^{j}(0) \bar{V}^{h}(0) \bar{V}^{k}(0) \bar{V}^{l}(0) \rho_{h, j}^{Z} \rho_{l, k}^{Z} \frac{\left(T_{h} \wedge T_{j} \wedge T_{l} \wedge T_{k}-T_{i}\right)}{T_{n}-T_{i}} v_{j} v_{k} \rho_{j, k}^{V}} \\
v^{Y}(t) & =\sqrt{\sum_{h, j, k, l=i+1}^{n} \bar{V}^{j}(0) \bar{V}^{h}(0) \bar{V}^{k}(0) \bar{V}^{l}(0) \rho_{h, j}^{Z} \rho_{l, k}^{Z} v_{j} v_{k} 1_{\left\{t<T_{h} \wedge T_{j} \wedge T_{l} \wedge T_{k}\right\}} \rho_{j, k}^{V}} \\
\hat{V}^{j}(t) & =\frac{V^{j}(t)}{V^{Y}} \\
\rho & =\frac{\sqrt{\sum_{h, j, k=i+1}^{n} \bar{V}^{j}(0) \bar{V}^{h}(0) \bar{V}^{k}(0) \rho_{h, j}^{Z} v_{j} \rho_{j, k}^{V}}}{v}
\end{aligned}
$$

We have been able to compute the forward SABR dynamic of the forward ratio, at time $T_{i}$, we can rewrite the payoff as follows

$$
\begin{aligned}
N\left[w\left(\alpha \frac{I\left(T_{n}\right)}{I\left(T_{i}\right)}-1-k\right)^{+}\right] & =\alpha N\left[w\left(\frac{I\left(T_{n}\right)}{I\left(T_{i}\right)}-\frac{1+k}{\alpha}\right)^{+}\right] \\
& =N^{1}\left[w\left(\frac{I\left(T_{n}\right)}{I\left(T_{i}\right)}-1-k^{1}\right)^{+}\right] \\
& =N^{1}\left[w\left(Y^{n, i}\left(T_{n}\right)-1-k^{1}\right)^{+}\right]
\end{aligned}
$$

\subsection{Real swaption and payoff transformation}

Given those SABR parameters, we have the real swaption which can be seen in first approximation as a zero coupon forward start option. The payoff is the following at $T_{i}$ expiry date of the option:

$$
\begin{aligned}
& N\left[w\left(\frac{I\left(T_{n}\right)}{I\left(T_{i}\right)}(1+k)^{n-i}-\prod_{j=i^{*}+1}^{n^{*}}\left(1+\delta^{j} L_{j}^{\delta}\right)\right)^{+}\right] \\
& =N\left[w\left(\frac{I\left(T_{n}\right)}{I\left(T_{i}\right)}(1+k)^{n-i}\right)-\prod_{j=i^{*}+1}^{n^{*}}\left(1+\delta^{j} L_{j}^{\delta}\right)^{+}\right]
\end{aligned}
$$




$$
\begin{aligned}
& =N\left[w\left(\left(\frac{I\left(T_{n}\right)}{I\left(T_{i}\right)}(1+k)^{n-i}\left(-\frac{1}{P\left(T_{i}, T_{n}\right)}\right)^{+}\right)\right]\right. \\
& =(1+k)^{n-i} N\left[w\left(\frac{I\left(T_{n}\right)}{I\left(T_{i}\right)}-\frac{1}{P\left(T_{i}, T_{n}\right)(1+k)^{n-i}}\right)^{+}\right] \\
& =(1+k)^{n-i} N\left[w\left(\frac{I\left(T_{n}\right)}{I\left(T_{i}\right)}-\frac{B A\left(T_{i}, T_{n}\right)}{(1+k)^{n-i}}\right)^{+}\right]
\end{aligned}
$$

we can also consider a first order approximation for the bank account (indeed by ignoring its volatility).

$$
\begin{aligned}
& N\left[w\left(\frac{I\left(T_{n}\right)}{I\left(T_{i}\right)}(1+k)^{n}-B A\left(T_{i}, T_{n}\right)\right)^{+}\right] \\
& =(1+k)^{n-i} N\left[w\left(\frac{I\left(T_{n}\right)}{I\left(T_{i}\right)}-\mathrm{E}_{t}^{T_{n}}\left[B A\left(T_{i}, T_{n}\right)\right] \frac{1}{(1+k)^{n-i}}\right)^{+}\right]
\end{aligned}
$$

By ignoring the volatility of the zero coupon, we miss-price the option, indeed the volatility of the spread can be lower or higher depending on the correlation between the forward ratio and the discrete bank account. The second order approximation considers the real swaption as a spread option between a forward ratio inflation index (dynamics derived in the previous section) and the discrete bank account. For the last one, we have the following dynamics under the $Q_{T_{n}}$ forward measure.

$$
\begin{aligned}
d P_{t}\left(T_{i}, T_{N}\right)= & P\left(T_{i}, T_{n}\right)\left[\sum_{j=i^{*}+1}^{n^{*}}-\frac{\delta^{j}}{1+\delta^{j} L_{j}} d W^{j}+\frac{1}{2} \sum_{j=i^{*}+1}^{n^{*}} \frac{\left(\sigma^{j} \delta^{j} L_{j}\right)^{2}}{\left(1+\delta^{j} L_{j}\right)^{2}} d t\right] \\
& +P\left(T_{i}, T_{n}\right)\left[2 \sum_{i^{*}+1 \leq j<k \leq n^{*}} \frac{\sigma^{j} \delta^{j} L_{j}}{1+\delta^{j} L_{j}} \frac{\sigma^{k} \delta^{k} L_{j}}{1+\delta^{k} L_{k}} \rho_{j, k}^{r} d t\right] \\
d B A\left(T_{i}, T_{n}\right)= & B A\left(T_{i}, T_{n}\right)\left[\sum_{j=i^{*}+1}^{n^{*}} \frac{\delta_{j}}{1+\delta^{j} L_{j}} L_{j} \sigma^{j} d W^{j}\right]
\end{aligned}
$$

\subsubsection{No strike case}

In case of no strike, we can use a classical closed form for the spread option with no strike under Black and Scholes assumption. 


$$
\begin{aligned}
\text { SpreadOption }\left(T, K=0 ; s_{1}, s_{2}, \sigma_{1}, \sigma_{2}\right) & =\mathrm{E}_{t}^{T_{n}}\left[w\left(S_{T}^{1}-S_{T}^{2}\right)^{+}\right] \\
& =s^{2} B S\left(T, 1 ; \frac{s_{1}}{s_{2}}, \tilde{\sigma}:=\sqrt{\sigma_{1}^{2}-2 \rho \sigma_{1} \sigma_{2}+\sigma_{2}^{2}}\right) \\
s_{1} & :=E_{t}^{T_{n}}\left[\frac{I_{n}^{f}\left(T_{n}\right)}{I_{i}^{f}\left(T_{i}\right)}\right](1+k)^{n-i} \\
s_{2} & :=E_{t}^{T_{n}}\left[B A\left(T_{i}, T_{n}\right)\right]
\end{aligned}
$$

We use a freezing technique to compute the volatility of the bank account. What can we do in the non-null strike case?

\subsubsection{Strike case}

In this section, we will consider the following payoff:

$$
\text { SpreadOption }^{\alpha, \beta, w}(T, K)=\mathrm{E}_{t}^{T_{n}}\left[w\left(\alpha \frac{I\left(T_{n}\right)}{I\left(T_{i}\right)}+\beta B A\left(T_{i}, T_{n}\right)-K\right)^{+}\right]
$$

The pricing of this option will need more works as the change of numeraire technique can not be applied. We proposed the following numerical methods:

- moment matching

- integration

- normal proxy.

The methods above will be described in Appendix.

\section{Conclusions}

We derive analytical proxy for forward volatility of the ZC swaption and real rate option. We extend Mercurio and Moreni (2010) LMM SABR in order to price the forward starting inflation option. We have shown the link between this option and the real rate swaption. After deriving the equation under LMM-SABR, we decide to approximate the bank account by a lognormal process via a freezing method. The result of our assumption is that the pricing of the real swaption can be interpreted as a spread option between a bank account weighted and forward ratio inflation index. We have implemented different types of correlation parametrisation and two types of proxies:

- $\quad$ one ignoring the bank account volatility, e.g., real swaption is seen as a forward starting inflation index zero coupon option with a strike corresponding to the forward bank account (or invert of the forward zero coupon)

- one considering the bank account as lognormal process thanks to a freezing method e.g., the real swaption is seen as a spread option. 
We give to the user indication of the mathematical consistent value which can be perturbed to match market quotes. However, care must be taken with the correlation parametrisation as a projection algorithm has to be applied.

The calibration process subjectivity - which market information should be the most relevant in our pricing framework - should reflect our hedging strategy. Indeed, if the YoY volatility is already a forward variance information, the spread of ZC swap can also capture the forward variance of the $\mathrm{ZC}$ swaption.

\section{References}

Belgrade, N., Benhamou, E. and Koehler, E. (2004) 'A market model for inflation', Working paper. Hinnerich, M. (2006) Inflation Indexed Swaps and Swaptions, Working paper.

Kenyon, C. (2006) 'Inflation is normal', Risk Magazine.

Mercurio, F. and Moreni, N. (2006) 'Inflation with a smile', Risk Magazine.

Mercurio, F. and Moreni, N. (2010) 'A multi-factor SABR model for forward inflation rate', Risk Magazine.

Merton, R.C. (1976a) 'Theory of rational option pricing', Journal of Economics and Management Science, pp.141-183.

Wimmer, H. (2006) Libor Market Models and Inflation Derivatives, Technische Universitat Munchen.

\section{Appendix}

Forward inflation index ratio dynamics under the $Q_{T}$-forward measure under the Mercurio SABR model

00.00 .0000

$$
\begin{aligned}
d I_{i}^{f}(t) & =I_{i}^{f}\left[\sum_{j=\beta(t)}^{i} V^{j}(t) d Z_{t}^{j, i}\right] \\
d I_{i-1}^{f}(t) & =I_{i-1}^{f}\left[-\frac{\delta L^{i}(t)}{1+\delta L^{i}(t)} \sigma_{t}^{r, i} \sum_{j=\beta(t)}^{i-1} V^{j}(t) \rho_{i, j}^{F, Z} d t+\sum_{j=\beta(t)}^{i-1} V^{j}(t) d Z_{t}^{j, i-1}\right] \\
d V^{j}(t) & =V^{j}(t)\left[v^{j} d W_{t}^{j}\right] \\
d L^{j}(t) & =F^{j}(t) \sigma_{t}^{r, i} d W_{t}^{F, i}
\end{aligned}
$$

where both $Z_{t}^{I, i}$ and $Z_{t}^{I, i-1}$ are Brownian motion under the $Q^{T}$-forward measure. By a classical freezing drift method, we have the forward inflation index ratio which can be rewritten as follows. 


$$
\begin{aligned}
& d X_{t}=d\left(\frac{I_{i}^{f}(t)}{I_{i-1}^{f}(t)}\right) \\
& =\frac{I_{i}^{f}(t)}{I_{i-1}^{f}(t)}\left[\sum_{j=\beta(t)}^{i} V^{j}(t) d Z_{t}^{j, i}-\left[-\frac{\delta L^{i}(t)}{1+\delta L^{i}(t)} \sigma_{t}^{r, i} \sum_{j=\beta(t)}^{i-1} V^{j}(t) \rho_{i, j}^{F, Z} d t\right.\right. \\
& \left.\left.+\sum_{j=\beta(t)}^{i-1} V^{j}(t) d Z_{t}^{j, i-1}\right]\right] \\
& +\frac{I_{i}^{f}(t)}{I_{i-1}^{f}(t)}\left[\sum_{j=\beta(t)}^{i-1} \sum_{k=\beta(t)}^{i-1} V^{j}(t) V^{k}(t) \rho_{j, k}^{Z} d t\right. \\
& \left.-\sum_{j=\beta(t)}^{i} \sum_{k=\beta(t)}^{i-1} V^{j}(t) V^{k}(t) \rho_{j, k}^{Z} d t\right] \\
& =\frac{I_{i}^{f}(t)}{I_{i-1}^{f}(t)}\left[\frac{\delta L^{i}(t)}{1+\delta L^{i}(t)} \sigma_{t}^{r, i} \sum_{j=\beta(t)}^{i-1} V^{j}(t) \rho_{i, j}^{F, Z} d t-V^{i}(t)\right. \\
& \left.\sum_{k=\beta(t)}^{i-1} V^{k}(t) \rho_{j, k}^{Z} d t+V^{i}(t) d Z_{t}^{i, i}\right] \\
& d \ln \left(X_{t}\right)=\left[\frac{\delta L^{i}(t)}{1+\delta L^{i}(t)} \sigma_{t}^{r, i} \sum_{j=\beta(t)}^{i-1} V^{j}(t) \rho_{i, j}^{F, Z} d t-V^{i}(t)\right. \\
& \left.\sum_{k=\beta(t)}^{i-1} V^{k}(t) \rho_{j, k}^{Z} d t-\frac{V^{i}(t)^{2}}{2} d t\right]+V^{i}(t) d Z_{t}^{i, i} \\
& E_{t}^{T_{i}}\left[\frac{I_{i}^{f}\left(T_{i}\right)}{I_{i-1}^{f}\left(T_{i-1}\right)}\right]=\frac{I_{i}^{f}(t)}{I_{i-1}^{f}(t)} e^{D(t)} \\
& D(T)=\int_{0}^{T} \frac{\delta L^{i}(t)}{1+\delta L^{i}(t)} \sigma_{t}^{r, i} \sum_{j=\beta(t)}^{i-1} V^{j}(t) \rho_{i, j}^{F, Z} d t-V^{i}(t) \sum_{k=\beta(t)}^{i-1} V^{k}(t) \rho_{j, k}^{Z} d t \\
& \hat{D}(T)=\int_{0}^{T} \frac{\delta L^{i}(0)}{1+\delta L^{i}(0)} \sigma_{t}^{r, i} \sum_{j=\beta(t)}^{i-1} V^{j}(0) \rho_{i, j}^{F, Z} d t-V^{i}(0) \sum_{k=\beta(t)}^{i-1} V^{k}(0) \rho_{j, k}^{Z} d t
\end{aligned}
$$

Proxy computation for ZCII option in SABR LMM model

$$
\begin{aligned}
& d I_{i}^{f}(t)=I_{i}^{f}\left[\sum_{j=\beta(t)}^{i} V^{j}(t) d Z_{t}^{j, i}\right] \\
& d V^{j}(t)=V^{j}(t)\left[v^{j} d W_{t}^{j}\right] \\
& \rho_{V, Z}^{i, j} d t=d W_{t}^{j} d Z_{t}^{j, i}
\end{aligned}
$$

we would like to describe the $I\left(T^{n}\right)$ as a SABR: 


$$
\begin{aligned}
d I(t) & =I(t)\left[V d Z_{t}\right] \\
d V(t) & =V(t)\left[v d W_{t}\right] \\
\rho d t & =d W_{t} d Z_{t}
\end{aligned}
$$

In order to find $V_{0}$ we match the quadratic variation of $I_{t}^{n}$

$$
\begin{aligned}
\frac{d<I^{n}>t}{I_{t}^{n}} & =V(t)^{2} d t \\
& =\sum_{j, h=\beta(t)}^{n} \rho_{i, h}^{Z} V^{j}(t) V^{h}(t) d t \\
& \Rightarrow V(0)=\sum_{j, h=\beta(t)}^{n} \rho_{i, h}^{Z} V^{j}(0) V^{h}(0)
\end{aligned}
$$

this is the first proxy. By considering

$$
\begin{aligned}
\mathrm{E}\left[\frac{1}{T_{n}} \int_{0}^{T_{n}} V^{2} d t\right] & =V^{2}(0)\left[\frac{e^{\nu^{2} T_{n}}-1}{v^{2} T_{n}}\right] \\
\lim _{v \rightarrow 0} V^{2}(0)\left[\frac{e^{\nu^{2} T_{n}}-1}{v^{2} T_{n}}\right] & =V^{2}(0)
\end{aligned}
$$

In another hand, we have thanks to (1)

$$
\begin{aligned}
\mathrm{E}\left[\frac{1}{T_{n}} \int_{0}^{T_{n}} V^{2} d t\right] & =\mathrm{E}\left[\frac{1}{T_{n}} \int_{0}^{T_{n}} \sum_{j, h=\beta(t)}^{n} \rho_{i, h}^{Z} V^{j}(t) V^{h}(t) d t\right] \\
& =\mathrm{E}\left[\frac{1}{T_{n}} \int_{0}^{T_{n}} \sum_{j, h=1}^{n} \rho_{i, h}^{Z} V^{j}(t) V^{h}(t) 1_{\left\{t<T_{j} \wedge T_{h}\right\}} d t\right] \\
& =\frac{1}{T_{n}} \sum_{j, h=1}^{n} \int_{0}^{T_{n}} \rho_{i, h}^{Z} \mathrm{E}\left[V^{j}(t) V^{h}(t)\right] 1_{\left\{t<T_{j} \wedge T_{h}\right\}} d t \\
& =\frac{1}{T_{n}} \sum_{j, h=1}^{n} \int_{0}^{T_{j} \wedge T_{h}} \rho_{i, h}^{Z} \mathrm{E}\left[V^{j}(t) V^{h}(t)\right] d t \\
& =\frac{1}{T_{n}} \sum_{j, h=1}^{n} \int_{0}^{T_{j} \wedge T_{h}} \rho_{i, h}^{Z} V^{j}(0) V^{h}(0) e^{\rho_{j}, h v_{j} v_{h} t} d t \\
& =\frac{1}{T_{n}} \sum_{j, h=1}^{n} \rho_{i, h}^{Z} V^{j}(0) V^{h}(0)\left(\frac{e^{\rho_{j, h}, h v_{h} v_{h}\left(T_{j} \wedge T_{h}\right)}-1}{\rho_{j, h} v_{j} v_{h}}\right)
\end{aligned}
$$




$$
\begin{aligned}
\lim _{v \rightarrow 0} V^{2}(0)\left[\frac{e^{v h 2 T_{n}}-1}{v^{2} T_{n}}\right] & =\lim _{v_{1, \ldots}, v_{i} \rightarrow 0} \frac{1}{T_{n}} \sum_{j, h=1}^{n} \rho_{i, h}^{Z} V^{j}(0) V^{h}(0)\left(\frac{e^{\rho_{j, h} v_{j} v_{h}\left(T_{j} \wedge T_{h}\right)}-1}{\rho_{j, h} v_{j} v_{h}}\right) \\
& =\sum_{j, h=1}^{n} \rho_{i, h}^{Z} V^{j}(0) V^{h}(0) \frac{T_{j} \wedge T_{h}}{T_{n}} \\
V^{2}(0) & =\sum_{j, h=1}^{n} \rho_{i, h}^{Z} V^{j}(0) V^{h}(0) \frac{T_{j} \wedge T_{h}}{T_{n}}
\end{aligned}
$$

To retrieve a vol of vol proxy $v$

$$
\begin{aligned}
d V & =d\left(\sqrt{\sum_{j, h=\beta(t)}^{n} \rho_{j, h}^{Z} V^{j}(t) V^{h}(t)}\right) \\
& =\frac{1}{2 V} d\left(\sum_{j, h=\beta(t)}^{n} \rho_{i, h}^{Z} V^{j}(t) V^{h}(t)\right) \\
& =\frac{1}{2 V} d\left(\sum_{j, h=1}^{n} \rho_{j, h}^{Z} V^{j}(t) V^{h}(t) 1_{\left\{t<T_{j} \wedge T_{h}\right\}}\right) \\
& =\frac{1}{2 V} \sum_{j, h=1}^{n} \rho_{j, h}^{Z} d\left(V^{j}(t) V^{h}(t) 1_{\left\{t<T_{j} \wedge T_{h}\right\}}\right) \\
& =\frac{1}{2 V} \sum_{j, h=1}^{n} \rho_{j, h}^{Z} V^{j}(t) V^{h}(t)\left[v_{j} d W_{j}+v_{h} d W_{h}\right] 1_{\left\{t<T_{j} \wedge T_{h}\right\}}+\ldots d t \\
& =V \sum_{j, h=1}^{n} 1_{\left\{t<T_{j} \wedge T_{h}\right\}} \rho_{j, h}^{Z} \hat{V}^{j}(t) \hat{V}^{h}(t) v_{j} d W_{j}+\ldots d t \\
d Z_{t} & =\sum_{j=1}^{n} \frac{V_{j}}{V} d Z_{j}^{n} \\
& =\sum_{j=1}^{n} \bar{V}^{j}(t) 1_{\left\{t<T_{j}\right\}} d Z_{j}^{n}
\end{aligned}
$$

So

$$
\begin{aligned}
d<V>_{t} & =V^{2} \sum_{j, h, k, l=1}^{n} 1_{\left\{t<T_{j} \wedge T_{h} \wedge T_{k} \wedge T_{l}\right\}} \rho_{j, h}^{Z} \rho_{k, l}^{Z} \bar{V}^{j}(t) \bar{V}^{h}(t) v_{j} \bar{V}^{k}(t) \bar{V}^{l}(t) v_{k} \rho_{j, k} d t \\
& =V^{2} v
\end{aligned}
$$

using a classical freezing method, we can deduce this two following proxy for $v$ : 


$$
\begin{aligned}
V & =\sqrt{\sum_{j, h, k, l=1}^{n} 1_{\left\{t<T_{j} \wedge T_{h} \wedge T_{k} \wedge T_{l}\right\}} \rho_{j, h}^{Z} \rho_{k, l}^{Z} \bar{V}^{j}(0) \bar{V}^{h}(0) v_{j} \bar{V}^{k}(0) \bar{V}^{l}(0) v_{k} \rho_{j, k}} \\
v & =\sqrt{\sum_{j, h, k, l=1}^{n} \rho_{j, h}^{Z} \rho_{k, l}^{Z} \bar{V}^{j}(0) \bar{V}^{h}(0) v_{j} \bar{V}^{k}(0) \bar{V}^{l}(0) v_{k} \rho_{j, k} \frac{T_{j} \wedge T_{h} \wedge T_{k} \wedge T_{l}}{T_{n}}} \\
v_{0} & =\sum_{j, h=1}^{n} \rho_{j, h}^{Z} \rho_{j, h}^{Z} \bar{V}^{j}(0) \bar{V}^{h}(0) \frac{T_{j} \wedge T_{h}}{T_{n}}
\end{aligned}
$$

$v_{0}$ is a proxy from our first computation of the quadratic variation of the variance. Given $\nu$, we want to be able to approximate $\rho$

$$
\begin{aligned}
d<V, Z>_{t} & =<V \sum_{j, h=1}^{n} 1_{\left\{t<T_{j} \wedge T_{h}\right\}} \rho_{j, h}^{Z} \widehat{V}^{j}(t) \widehat{V}^{h}(t) v_{j} d W_{j}, \sum_{j=1}^{n} \widehat{V}^{j}(t) 1_{\left\{t<T_{j}\right\}} d Z_{j}^{n}> \\
& =V \sum_{j, h, l=1}^{n} 1_{\left\{1<T_{j} \wedge T_{h} \wedge T_{l}\right\}} \rho_{j, h}^{Z} \bar{V}^{j}(t) \bar{V}^{h}(t) v_{j} \bar{V}^{l}(t) 1_{\{t<\}} \rho_{j, l}^{Z, W} d t \\
& =\rho V v d t
\end{aligned}
$$

So, we can deduce $\rho$ as function of ours proxies for $V_{0}$ and $v$ :

$$
\begin{gathered}
\rho:=\frac{V_{0} \sum_{j, h, l=1}^{n} \rho_{j, h}^{Z} \hat{V}^{j}(0) \hat{V}^{h}(0) v_{j} \hat{V}_{l}(0) \rho_{j, l}^{Z, W} \frac{T_{j} \wedge T_{h} \wedge T_{l}}{T_{n}}}{V_{0} \sum_{j, h=1}^{n} \rho_{j, h}^{Z} \rho_{j, h}^{Z} \bar{V}^{j}(0) \bar{V}^{h}(0) \frac{T_{j} \wedge T_{h}}{T_{n}}} \\
\simeq \frac{\sum_{j, h, l=1}^{n} \rho_{j, h}^{Z} \bar{V}^{j}(0) \bar{V}^{h}(0) v_{j} \bar{V}^{l}(0) 1_{\left\{t<T_{j} \wedge T_{h} \wedge T\right\}} \rho_{j, l}^{Z, W}}{\sum_{j, h=1}^{n} \rho_{j, h}^{Z} \rho_{j, h}^{Z} \bar{V}^{j}(0) \bar{V}^{h}(0)}
\end{gathered}
$$

\section{Proxy computation for real swaption in SABR LMM model}

As described in the section, the real swaption can be considered as a forward start zero coupon inflation indexed option, we will derive the equation which allows us to determine a consistent dynamic of the forward ration inflation index $\frac{I\left(T_{n}\right)}{I\left(T_{i}\right)}$ first of all, we have to determine the convexity adjustment of the ratio: 


$$
\begin{aligned}
& d X_{t}^{i, n}=d\left(\frac{I_{n}^{f}(t)}{I_{i}^{f}(t)}\right) \\
&=\frac{I_{n}^{f}(t)}{I_{i}^{f}(t)}\left[\sum_{j=\beta(t)}^{n} V^{j}(t) d Z_{t}^{j, n}-\right. \\
& {\left[\sum_{l=i+1}^{n}-\frac{\delta L^{l}(t)}{1+\delta L^{L}(t)} \sigma_{t}^{r, l} \sum_{j=\beta(t)}^{i} V^{j}(t) \rho_{l, j}^{F, Z} d t\right.} \\
&\left.\left.+\sum_{j=\beta(t)}^{i} V^{j}(t) d Z_{t}^{j, n}\right]\right] \\
&+\frac{I_{n}^{f}(t)}{I_{i}^{f}(t)}\left[\sum_{j, k=\beta(t)}^{i} V^{j}(t) V^{k}(t) \rho_{j, k}^{Z} d t\right.\left.-\sum_{j=\beta(t)}^{n} \sum_{k=\beta(t)}^{i} V^{j}(t) V^{k}(t) \rho_{j, k}^{Z} d t\right] \\
&=\frac{I_{n}^{f}(t)}{I_{i}^{f}(t)}\left[\bar{D}_{n, i}(t) d t+\sum_{j=i+1}^{n} V^{j}(t) d Z_{t}^{j, n}\right] \\
& \bar{D}_{n, i}(t):=\sum_{l=i+1}^{n}\left[\frac{\delta L^{l}(t)}{1+\delta L^{l}(t)} \sigma_{t}^{r, l} \sum_{j=\beta(t)}^{i} V^{j}(t) \rho_{i, j}^{F, Z}+\sum_{k=\beta(t)}^{i} V^{l}(t) V^{k}(t) \rho_{j, l}^{Z}\right] \\
& D_{n, i}(T):=\sum_{l=i+1}^{n}\left[\frac{\delta L^{l}(0)}{1+\delta L^{l}(0)} \sigma_{t}^{r, l} \sum_{j=\beta(t)}^{i} V^{j}(0) \rho_{i, j}^{F, Z}+\sum_{k=\beta(t)}^{i} V^{l}(0) V^{k}(0) \rho_{j, l}^{Z}\right]
\end{aligned}
$$

This calculus should be adjusted in case where the underlying rate for instance three months Libor has not the same frequency to the market observed inflation index for instance year on year.

$$
\begin{aligned}
& d X_{t}^{i, n}=d\left(\frac{I_{n}^{f}(t)}{I_{i}^{f}(t)}\right) \\
&=\frac{I_{n}^{f}(t)}{I_{i}^{f}(t)}\left[\sum_{j=\beta(t)}^{n} V^{j}(t) d Z_{t}^{j, n}-\left[\sum_{l=i^{*}+1}^{n^{*}}-\frac{\delta L^{l}(t)}{1+\delta L^{l}(t)} \sigma_{t}^{r, l} \sum_{j=\beta(t)}^{i} V^{j}(t) \rho_{l, j}^{F, Z} d t\right.\right. \\
&\left.\left.+\sum_{j=\beta(t)}^{i} V^{j}(t) d Z_{t}^{j, n}\right]\right]+\frac{I_{n}^{f}(t)}{I_{i}^{f}(t)}\left[\sum_{j, k=\beta(t)}^{i} V^{j}(t) V^{k}(t) \rho_{j, k}^{Z} d t\right. \\
&\left.\quad-\sum_{j=\beta(t)}^{n} \sum_{k=\beta(t)}^{i} V^{j}(t) V^{k}(t) \rho_{j, k}^{Z} d t\right] \\
&=\frac{I_{n}^{f}(t)}{I_{i}^{f}(t)}[\left.\bar{D}_{n, i}(t) d t+\sum_{j=i+1}^{n} V^{j}(t) d Z_{t}^{j, n}\right]
\end{aligned}
$$




$$
\begin{aligned}
\bar{D}_{n, i}(t): & \sum_{l=i^{*}+1}^{n^{*}} \frac{\delta L^{l}(t)}{1+\delta L^{l}(t)} \sigma_{t}^{r, l} \sum_{j=\beta(t)}^{i} V^{j}(t) \rho_{i, j}^{F, Z} \\
& +\sum_{l=i+1}^{n} \sum_{k=\beta(t)}^{i} V^{l}(t) V^{k}(t) \rho_{k, l}^{Z} \\
D_{n, i}(T): & =\sum_{l=i^{*}+1}^{n^{*}} \frac{\delta L^{l}(0)}{1+\delta L^{l}(0)} \sigma_{t}^{r, l} \sum_{j=\beta(t)}^{i} V^{j}(0) \rho_{i, j}^{F, Z}+\sum_{l=i+1}^{n} \sum_{k=\beta(t)}^{i} V^{l}(0) V^{k}(0) \rho_{j, l}^{Z}
\end{aligned}
$$

First, we consider the following process $Y_{t}^{n, i}$ :

$$
\begin{aligned}
& \frac{d Y_{t}^{n, i}}{Y_{t}^{n, i}}=\sum_{j=i+1}^{n} V^{j}(t) d Z_{t}^{j, n} \\
& Y_{0}^{n, i}=\frac{I_{n}^{f}(0)}{I_{i}^{f}(0)} e^{D_{n, i}(0)}
\end{aligned}
$$

then we want to find the associated SABR $\bar{Y}^{n, i}$ such that

$$
\begin{aligned}
\frac{d \bar{Y}_{\bar{Y}}^{n, i}}{\bar{Y}_{t}^{n, i}} & =V^{Y} d Z_{t} \\
\bar{Y}_{0}^{n, i} & =Y_{0}^{n, i} \\
d V^{Y} & =v^{Y} V^{Y} d W_{t} \\
\rho^{Y} d t & =d<W, Z>_{t} \\
V^{Y} & =\sqrt{\sum_{j, h=i+1}^{n} \rho_{i, h}^{Z} V^{j}(t) V^{h}(t) 1_{\left\{t<T_{j} \wedge T_{h}\right\}}} \\
d Z_{t} & =\sum_{j=i+1}^{n} \frac{V_{j}}{V^{Y}} d Z_{j}^{n} \\
& =\sum_{j=i+1}^{n} \hat{V}^{j}(t) 1_{\left\{t<T_{j}\right\}} d Z_{j}^{n}
\end{aligned}
$$

To retrieve the SABR parameters, we will as before workout the matching of the quadratic variation

$$
\begin{aligned}
\frac{d<\bar{Y}_{t}^{n, i}>_{t}}{\bar{Y}_{t}^{n, i}} & =V^{Y}(t)^{2} d t \\
& =\sum_{j, h=i+1}^{n} \rho_{j, h}^{Z} V^{j}(t) V^{h}(t) d t
\end{aligned}
$$

In another hand, we have thanks to (1) 


$$
\begin{aligned}
& \mathrm{E}\left[\frac{1}{\left(T_{n}-T_{i}\right)} \int_{T_{i}}^{T_{n}} V^{Y}(t)^{2} d t\right]=\mathrm{E}\left[\frac{1}{\left(T_{n}-T_{i}\right)} \int_{T_{i}}^{T_{n}} \sum_{j, h=i+1}^{n} \rho_{i, h}^{Z} V^{j}(t) V^{h}(t) d t\right] \\
& =\mathrm{E}\left[\frac{1}{\left(T_{n}-T_{i}\right)} \int_{T_{i}}^{T_{n}} \sum_{j, h=i+1}^{n} \rho_{i, h}^{Z} V^{j}(t) V^{h}(t) 1_{\left\{t<T_{j} \wedge T_{h}\right\}} d t\right] \\
& =\frac{1}{\left(T_{n}-T_{i}\right)} \sum_{j, h=i+1}^{n} \int_{T_{i}}^{T_{n}} \rho_{i, h}^{Z} \mathrm{E}\left[V^{j}(t) V^{h}(t)\right] 1_{\left\{t<T_{j} \wedge T_{h}\right\}} d t \\
& =\frac{1}{\left(T_{n}-T_{i}\right)} \sum_{j, h=i+1}^{n} \int_{T_{i}}^{T_{j} \wedge T_{h}} \rho_{i, h}^{Z} \mathrm{E}\left[V^{j}(t) V^{h}(t)\right] d t \\
& =\frac{1}{\left(T_{n}-T_{i}\right)} \sum_{j, h=i+1}^{n} \int_{T_{i}}^{T_{j} \wedge T_{h}} \rho_{i, h}^{Z} V^{j}(0) V^{h}(0) e^{\rho_{j, h} v_{j} v_{h} t} d t
\end{aligned}
$$

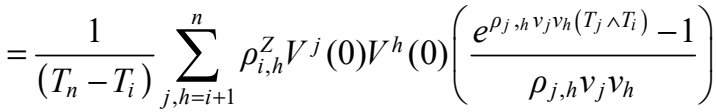

$$
\begin{aligned}
& \lim _{v \rightarrow 0} V^{Y_{2}}(0)\left[\frac{e^{v^{2}\left(T_{n}-T_{i}\right)}-1}{v^{2}\left(T_{n}-T_{i}\right)}\right]=\lim _{v 1, \ldots, v_{i} \rightarrow 0} \\
& \left\{\frac{1}{\left(T_{n}-T_{i}\right)} \sum_{j, h=i+1}^{n} \rho_{i, h}^{Z} V^{j}(0) V^{h}(0)\left(\frac{e^{\rho_{j, h} v_{j} v_{h}\left(T_{j} \wedge T_{i}\right)}-1}{\rho_{j, h} v_{j} v_{h}}\right)\right\} \\
& =\sum_{j, h=i+1}^{n} \rho_{i, h}^{Z} V^{j}(0) V^{h}(0) \frac{T_{j} \wedge T_{h}-T_{i}}{T_{n}-T_{i}} \\
& V^{Y^{2}}(0)=\sum_{j, h=i+1}^{n} \rho_{i, h}^{Z} V^{j}(0) V^{h}(0) \frac{T_{j} \wedge T_{h}-T_{i}}{T_{n}-T_{i}}
\end{aligned}
$$

To retrieve a vol of vol proxy $v$

$$
\begin{aligned}
d V^{Y} & =d\left(\sqrt{\sum_{j, h=l+1}^{n} \rho_{j, h}^{Z} V^{j}(t) V^{h}(t)}\right) \\
& =\frac{1}{2 V^{Y}} d\left(\sum_{j, h=t+1}^{n} \rho_{i, h}^{Z} V^{j}(t) V^{h}(t)\right)+\ldots d t \\
& =\frac{1}{2 V} d\left(\sum_{j, h=i+1}^{n} \rho_{j, h}^{Z} V^{j}(t) V^{h}(t) 1_{\left\{t<T_{j} \wedge T_{h}\right\}}\right)+\ldots d t
\end{aligned}
$$




$$
\begin{aligned}
& =\frac{1}{2 V^{Y}} \sum_{j, h=i+1}^{n} \rho_{j, h}^{Z} d\left(V^{j}(t) V^{h}(t) 1_{\left\{t<T_{j} \wedge T_{h}\right\}}\right)+\ldots d t \\
& =\frac{1}{2 V^{Y}} \sum_{j, h=i+1}^{n} \rho_{j, h}^{Z} V^{j}(t) V^{h}(t)\left[v_{j} d W_{j}+v_{h} d W_{h}\right] 1_{\left\{t<T_{j} \wedge T_{h}\right\}}+\ldots d t \\
& =V^{Y} \sum_{j, h=i+1}^{n} 1_{\left\{t<T_{j} \wedge T_{h}\right\}} \rho_{j, h}^{Z} \hat{V}^{j}(t) \hat{V}^{h}(t) v_{j} d W_{j}+\ldots d t \\
d<V^{Y}>_{t} & =V^{Y_{2}} \sum_{j, h, k, l=i+1}^{n} 1_{\left\{t<T_{j} \wedge T_{h} \wedge T_{k} \wedge T_{l}\right\}} \rho_{j, h}^{Z} \rho_{k, l}^{Z} \hat{V}^{j}(t) \hat{V}^{h}(t) \hat{V}^{k}(t) \hat{V}^{l}(t) v_{k} \rho_{j, k} d t \\
& =V^{2} v
\end{aligned}
$$

By using a classical freezing method, we can deduce this two following proxies for $v$ :

$$
\begin{aligned}
& V=\sqrt{\sum_{j, h, k, l=i+1}^{n} 1_{\left\{t<T_{j} \wedge T_{h} \wedge T_{k} \wedge T_{l}\right\}} \rho_{j, h}^{Z} \rho_{k, l}^{Z} \hat{V}^{j}(0) \hat{V}^{h}(0) v_{j} \hat{V}^{k}(0) \hat{V}^{l}(0) v_{k} \rho_{j, k}} \\
& v=\sqrt{\sum_{j, h, k, l=i+1}^{n} \rho_{j, h}^{Z} \rho_{k, l}^{Z} \bar{V}^{j}(0) \bar{V}^{h}(0) v_{j} \bar{V}^{k}(0) \bar{V}^{l}(0) v_{k} \rho_{j, k} \frac{T_{j} \wedge T_{h} \wedge T_{k} \wedge T_{l}-T_{i}}{T_{n}-T_{i}}} \\
& v_{0}=\sum_{j, h=i+1}^{n} \rho_{j, h}^{Z} \rho_{j, h}^{Z} \bar{V}^{j}(0) \bar{V}^{h}(0) \frac{T_{j} \wedge T_{h}-T_{i}}{T_{n}-T_{i}}
\end{aligned}
$$

$v_{0}$ is a proxy from our first computation of the quadratic variation of the variance. Given $\nu$, we want to be able to approximate $\rho$

$$
\begin{aligned}
d<V^{Y}, d Z>_{t}=<V^{Y} \sum_{j, h=i+1}^{n} 1_{\left\{t<T_{j} \wedge T_{h}\right\}} \rho_{j, h}^{Z} \bar{V}^{j}(t) \bar{V}^{h}(t) v_{j} d W_{j}, \sum_{j=1}^{n} \bar{V}^{j}(t) 1_{\left\{t<T_{j}\right\}} d Z_{j}^{n}> \\
=V^{Y} \sum_{j, h, l=i+1}^{n} 1_{\left\{1<T_{j} \wedge T_{h} \wedge T_{\}}\right\}} \rho_{j, h}^{Z} \bar{V}^{j}(t) \bar{V}^{h}(t) v_{j} \bar{V}^{l}(t) 1_{\{t<\}} \rho_{j, l}^{Z, W} d t \\
=\rho V^{Y} v d t
\end{aligned}
$$

Therefore, we can deduce $\rho$ as function of ours proxies for $V_{0}^{Y}$ and $v$ :

$$
\begin{gathered}
\rho:=\frac{V_{0}^{Y} \sum_{j, h, l=i+1}^{n} \rho_{j, h}^{Z} \hat{V}^{j}(0) \hat{V}^{h}(0) v_{j} \widehat{V}^{l}(0) \rho_{j, l}^{Z, W} \frac{T_{j} \wedge T_{h} \wedge T_{l}}{T_{n}-T_{i}}}{V_{0}^{Y} \sum_{j, h=i+1}^{n} \rho_{j, h}^{Z} \rho_{j, h}^{V} \bar{V}^{j}(0) \bar{V}^{h}(0) \frac{T_{j} \wedge T_{h}-T_{i}}{T_{n}-T_{i}}} \\
\simeq \frac{\sum_{j, h, l=i+1}^{n} \rho_{j, h}^{Z} \bar{V}^{j}(0) \bar{V}^{h}(0) v_{j} \bar{V}^{l}(0) 1_{\left\{t<T_{j} \wedge T_{h} \wedge T_{l}\right\}} \rho_{j, l}^{Z, W}}{\sum_{j, h=i+1}^{n} \rho_{j, h}^{Z} \rho_{j, h}^{Z} \hat{V}^{j}(0) \hat{V}^{h}(0)}
\end{gathered}
$$

In order to use our derivation adequately, we want to consider the following payoff representation in case of Libor market model: 


$$
\begin{aligned}
N\left[w\left(\frac{I\left(T_{n}\right)}{I\left(T_{i}\right)}(1+k)^{n-i}-\prod_{j=i+1}^{n-j}\left(1+\delta^{j} L_{j}^{\delta}\right)\right)^{+}\right] & =(1+k)^{n-i} N\left[w \left(\frac{I\left(T_{n}\right)}{I\left(T_{i}\right)}\right.\right. \\
& \left.\left.=\frac{1}{P\left(T_{i}-T_{n}\right)} \frac{1}{(1+k)^{n-i}}\right)^{+}\right] \\
\alpha & =1\left[w\left(\alpha \frac{I\left(T_{n}\right)}{I\left(T_{i}\right)}+\beta P\left(T_{i}, T_{n}\right)-K\right)^{+}\right] \\
\beta & =\frac{1}{(1+k)^{n-i}} \\
N^{1} & =(1+k)^{n-i} N
\end{aligned}
$$

We have a basket option between a forward bond and an inflation forward ratio.

\section{Moment matching}

Considering an option with the following payoff

$$
\left(\alpha A_{T_{n}}+\beta B_{T_{n}}-K\right)^{+}
$$

Basket: $\alpha \beta>0$

We define $S$ the process lognormal representing the basket as follows.

$$
\begin{aligned}
& S_{t}=\left(\alpha A_{t}+\beta B_{t}\right) \\
& d S_{t}=S_{t}\left(\sigma^{S} d W_{t}\right) \\
& S_{t_{0}}=s
\end{aligned}
$$

and we retrieve by matching the two first moments

$$
\begin{aligned}
\mathrm{E}_{t_{0}}^{T_{n}}\left[S_{T_{n}}\right] & =s \\
& =\alpha \mathrm{E}_{t_{0}}^{T_{n}}\left[A_{T_{n}}\right]+\beta \mathrm{E}_{t_{0}}^{T_{n}}\left[B_{T_{n}}\right] \\
& =\alpha A_{t_{0}}+\beta B_{t_{0}} \\
\sigma^{S^{2}} & =\frac{1}{T_{n}-t_{0}} \ln \left[\frac{E_{t_{0}}^{T_{n}}\left[\alpha A_{T_{n}}+\beta B_{t}\right]^{2}}{s^{2}}\right] \\
\mathrm{E}_{t_{0}}^{T_{n}}\left[\left(\alpha A_{T_{n}}+\beta B_{t}\right)^{2}\right] & =\alpha^{2} \mathrm{E}_{t_{0}}^{T_{n}}\left[A_{T_{n}}^{2}\right]+\beta^{2} \mathrm{E}_{t_{0}}^{T_{n}}\left[B_{T_{n}}^{2}\right]+2 \alpha \beta \mathrm{E}_{t_{0}}^{T_{n}}\left[B_{T_{n}} A T_{n}\right] \\
& =\alpha^{2} A_{t_{0}}^{2} e^{\sigma_{A}^{2}\left(T_{n}-t_{0}\right)}+\beta^{2} B_{t_{0}}^{2} \sigma^{\sigma_{B}^{2}\left(T_{n}-t_{0}\right)}+2 \alpha \beta B_{t_{0}} A_{t_{0}} e^{\sigma_{A} \sigma_{B} \bar{\rho}\left(T_{n}-t_{0}\right)}
\end{aligned}
$$

For our purposes, we will consider a freezing method to have $\sigma_{B}$ the vol of the bond and the SABR lognormal volatility at strike adjusted by the forward of the bond. The major issue is to approximate the $\rho$ parameter. 


$$
\bar{\rho}=\frac{\left\langle\sum_{j=i+1}^{n} V^{j}(t) d Z_{t}^{j, n}, \sum_{j=i^{*}+1}^{n^{*}} \frac{\delta L^{j} \sigma_{t}^{r, j}}{1+\delta L_{t}^{j}} d W^{r, j}\right\rangle}{\sqrt{\left\langle\sum_{j=i^{*}+1}^{n^{*}} \frac{\delta L^{j} \sigma_{t}^{r, j}}{1+\delta L_{t}^{j}} d W^{r, j}><\sum_{j=i+1}^{n} V^{j}(t) d Z_{t}^{j, n}\right\rangle}}
$$

Spread: $\alpha \beta<0$

The potential negativity of the spread push us to consider a three moments matching. We define $C$ a lognormal process and a constant process $D_{t}$ :

$$
\begin{aligned}
S_{t} & =\left(\alpha A_{t}+\beta B_{t}\right) \\
S_{t} & =C_{t}+D_{t} \\
d C_{t} & =C_{t}\left(\sigma^{B} d W_{t}\right) \\
C_{t_{0}} & =c \\
\forall t & >0, \\
D t & =d<0
\end{aligned}
$$

And, we retrieve the missing parameters by matching the three first moments

$$
\begin{aligned}
& \mathrm{E}_{t_{0}}^{T_{n}}\left[S_{T_{n}}\right]=c+d \\
& =\alpha \mathrm{E}_{t_{0}}^{T_{0}}\left[A_{T_{n}}\right]+\beta \mathrm{E}_{t_{0}}^{T_{n}}\left[B_{T_{n}}\right] \\
& =\alpha A_{t_{0}}+\beta B_{t_{0}} \\
& \mathrm{E}_{t 0}^{T_{n}}\left[S_{T_{n}}^{2}\right]=c^{2} e^{\sigma^{C^{2}}\left(T_{n}-t_{0}\right)}+2 c d+d^{2} \\
& \mathrm{E}_{t_{0}}^{T_{n}}\left[\left(\alpha A_{T_{n}}+\beta B_{t}\right)^{2}\right]=\alpha^{2} \mathrm{E}_{t_{0}}^{T_{n}}\left[A_{T_{n}}^{2}\right]+\beta^{2} \mathrm{E}_{t_{0}}^{T_{n}}\left[B_{T_{n}}^{2}\right]+2 \alpha \beta \mathrm{E}_{t_{0}}^{T_{n}}\left[B_{T_{n}} A T_{n}\right] \\
& =\alpha^{2} A_{t_{0}}^{2} e^{\sigma_{A}^{2}\left(T_{n}-t_{0}\right)}+\beta^{2} B_{t_{0}}^{2} e^{\sigma_{B}^{2}\left(T_{n}-t_{0}\right)}+2 \alpha \beta B_{t_{0}} A_{t_{0}} e^{\sigma_{A} \sigma_{B} \bar{\rho}\left(T_{n}-t_{0}\right)} \\
& \mathrm{E}_{t_{0}}^{T_{n}}\left[S_{T_{n}}^{3}\right]=c^{3} e^{3 \sigma^{C}}\left(T_{n}-t_{0}\right)+3 d c^{2} e^{\sigma^{C^{2}}\left(T_{n}-t_{0}\right)}+2 c d^{2}+d^{3} \\
& \mathrm{E}_{t_{0}}^{T_{n}}\left[\left(\alpha A_{T_{n}}+\beta B_{t}\right)^{3}\right]=\alpha^{3} \mathrm{E}_{t_{0}}^{T_{n}}\left[A_{T_{n}}^{3}\right]+\beta^{3} \mathrm{E}_{t_{0}}^{T_{n}}\left[B_{T_{n}}^{3}\right] \\
& +3 \alpha^{2} \beta \mathrm{E}_{t_{0}}^{T_{n}}\left[B_{T_{n}}\right] \mathrm{E}_{t_{0}}^{T_{n}}\left[A_{T_{n}}^{2}\right]+3 \alpha \beta^{2} \mathrm{E}_{t_{0}}^{T_{n}}\left[B_{T_{n}}^{3}\right] \mathrm{E}_{t_{0}}^{T_{n}}\left[A_{T_{n}}\right] \\
& =\alpha^{3} A_{t_{0}}^{3} e^{3 \sigma_{A}^{2}\left(T_{n}-t_{0}\right)}+\beta^{3} B_{t_{0}}^{3} e^{3 \sigma_{B}^{2}\left(T_{n}-t_{0}\right)} \\
& +3 \alpha^{2} \beta A_{t_{0}}^{2} B_{t_{0}} e^{\sigma_{A}^{2}\left(T_{n}-T_{0}\right)} e^{-2 \sigma_{A} \sigma_{B} \bar{\rho}\left(T_{n}-t_{0}\right)} \\
& +3 \alpha \beta^{2} A_{t_{0}} B_{t_{0}}^{2} e^{\sigma_{B}^{2}\left(T_{n}-t_{0}\right)} e^{-2 \sigma_{A} \sigma_{B} \bar{\rho}\left(T_{n}-t_{0}\right)}
\end{aligned}
$$

For our purposes, we will consider a freezing method to have $\sigma_{B}$ the vol of the discrete bank account and the SABR lognormal volatility at strike adjusted by the forward of the Bond. The major issue is to approximate the $\rho$ parameter. 


$$
\rho=\frac{\left\langle\sum_{j=i+1}^{n} V^{j}(t) d Z_{t}^{j, n}, \sum_{j=i+1}^{n^{*}} \frac{\delta L^{j} \sigma_{t}^{r, j}}{1+\delta L_{t}^{j}} d W^{r, j}>\right.}{\sqrt{<\sum_{j=i+1}^{n} \frac{\delta L^{j} \sigma_{t}^{r, j}}{1+\delta L_{t}^{j}} d W^{r, j}><\sum_{j=i+1}^{n} V^{j}(t) d Z_{t}^{j, n}>}}
$$

\section{Integration}

$$
\begin{aligned}
\mathrm{E}_{t_{0}}^{T_{n}}\left[\alpha A_{T_{n}}+\beta B_{T_{n}}-K\right] & =\mathrm{E}_{t_{0}}^{T_{n}}\left[\alpha A_{T_{n}}+\beta B_{T_{n}}-K \mid B_{T_{n}}\left(w^{B}\right)\right] \\
& =\int_{-\infty}^{+\infty} B S\left(\tilde{K}, T_{n}-T_{i} ; \bar{A}, \mu^{A}, \bar{\sigma}^{A}\right) f_{B(w)} d w \\
\tilde{K} & =K-\beta b e^{-\frac{\sigma B^{2}}{2}\left(T_{n}-T_{i}\right)+\sigma^{B} w} \\
\bar{\sigma}^{A^{2}} & =\sigma_{A}^{2}\left(1-\rho^{2}\right) \\
\sigma_{A} & =\sigma^{S A B R}(\tilde{K}) \\
\mu^{A^{2}} & =\left[-\frac{\sigma_{A}^{2}}{2}+\sigma_{A} \rho w+\frac{\bar{\sigma}^{A^{2}}}{2}\right]\left(T_{n}-T_{i}\right)
\end{aligned}
$$

The key point is that conditioned to the $w^{B}$ value $A$ is still a lognormal process.

\section{Normal proxy}

We convert the lognormal volatility to a normal one. Then, we can leave the correlation as a freedom for the broker to feet the market or calibrate it. We consider two processes $\tilde{A}$ and $\tilde{B}$ Gaussians:

$$
\begin{aligned}
\tilde{A}_{0} & =\mathrm{E}_{t_{0}}^{T_{n}}\left[A_{T_{n}}\right] \\
d \tilde{A}_{t} & =\tilde{\sigma}_{A} d W_{t}^{A} \\
\tilde{B}_{0} & =\mathrm{E}_{t_{0}}^{T_{n}}\left[B_{T_{n}}\right] \\
d \tilde{B}_{t} & =\tilde{\sigma}_{B} d W_{t}^{B}
\end{aligned}
$$

Therefore, the spread $\tilde{S}_{t}$ is Gaussian as well with:

$$
\begin{aligned}
\tilde{S}_{t} & =\alpha \tilde{A}_{t}+\beta \tilde{B}_{t} \\
\tilde{S}_{0} & =\alpha \tilde{A}_{0}+\beta \tilde{B}_{0} \\
\tilde{\sigma}_{S}^{2} & =\left[\alpha^{2} \tilde{\sigma}_{A}^{2}+\beta^{2} \tilde{\sigma}_{B}^{2}+2 \rho \tilde{\sigma}_{A} \tilde{\sigma}_{B}\right]
\end{aligned}
$$

The normal volatility is such that it match the actual lognormal price around the strike adjusted.

$$
B S\left(T, K^{x} ; x, \sigma_{X}\right)=\operatorname{Bachelier}\left(T, K^{x} ; x, \tilde{\sigma}_{X}\right)
$$


In our case, the strike adjusted is for $A: K^{A}:=K-\mathrm{E}_{t_{0}}^{T_{n}}\left[\beta B_{T_{n}}\right]$ and respectively for $B: K^{B}:=K-\mathrm{E}_{t_{0}}^{T_{n}}\left[\alpha A_{T_{n}}\right]$. As a reminder we derive the Bachelier option price formula:

$$
\begin{aligned}
\operatorname{Bachelier}\left(T, K ; x, \sigma_{X}\right) & =P(0, T)\left[(x-K) N(d)-\sigma_{X} \sqrt{T} \phi(d)\right] \\
d & =\frac{x-K}{\sigma_{X} \sqrt{T}}
\end{aligned}
$$

with $\phi$ the density of a normal 0,1 .

Zero coupon dynamic and discrete bank account LMM and under the $Q_{T_{n}}$ forward measure

By definition of a zero coupon $P\left(T_{i}, T_{n}\right):=\prod_{j=i^{*}+1}^{n^{*}} \frac{1}{\left(1+\delta^{j} L_{j}^{\delta}\right)}$

$$
\begin{aligned}
d P\left(T_{i}, T_{n}\right)= & \prod_{j=i^{*}+1}^{n^{*}} \frac{1}{\left(1+\delta^{j} L_{j}^{\delta}\right)} \\
= & P\left(T_{i}, T_{n}\right)\left[\sum_{j=i^{*}+1}^{n^{*}}-\frac{\delta^{j}}{1+\delta^{j} L_{j}} d L_{j}\right. \\
& \left.+\frac{1}{2} \sum_{k, j=i^{*}+1}^{n^{*}} \frac{\sigma^{i} \delta^{j} L_{j}}{1+\delta^{j} L_{j}} \frac{\sigma^{k} \delta^{k} L_{j}}{1+\delta^{k} L_{k}} \rho_{j, k}^{r} d t\right] \\
= & P\left(T_{i}, T_{n}\right)\left[\sum_{j=i^{*}+1}^{n^{*}}-\frac{\delta^{j}}{1+\delta^{j} L_{j}} L_{j} \sigma^{j}\left(d W^{r, j}-\sum_{k=j+1}^{n} \frac{\delta^{k} \sigma^{k} L_{k}}{1+\delta^{k} L_{k}} \rho_{j, k}^{r} d t\right)\right] \\
+ & P\left(T_{i}, T_{n}\right)\left[\frac{1}{2} \sum_{k, j=i^{*}+1}^{n^{*}} \frac{\sigma^{j} \delta^{j} L_{j}}{1+\delta^{j} L_{j}} \frac{\sigma^{k} \delta^{k} L_{j}}{1+\delta^{k} L_{k}} \rho_{j, k}^{r} d t\right] \\
= & P\left(T_{i}, T_{n}\right)\left[\sum_{k, j=i^{*}+1}^{n^{*}} \frac{\delta^{j}}{1+\delta^{j} L_{j}} L_{j} \sigma^{j} d W^{r, j}+\frac{1}{2} \sum_{j=i+1}^{n} \frac{\left(\sigma^{j} \delta^{j} L_{j}\right)}{\left(1+\delta^{j} L_{j}\right)} d t\right] \\
+ & P\left(T_{i}, T_{n}\right)\left[2 \sum_{i^{*}+1 \leq j<k \leq n^{*}} \frac{\sigma^{j} \delta^{j} L_{j}}{1+\delta^{j} L_{j}} \frac{\sigma^{k} \delta^{k} L_{j}}{1+\delta^{k} L_{k}} \rho_{j, k}^{r} d t\right]
\end{aligned}
$$

By definition of a zero coupon $B A\left(T_{i}, T_{n}\right):=\prod_{j=i^{*}+1}^{n^{*}}\left(1+\delta^{j} L_{j}^{\delta}\right)$ 


$$
\begin{aligned}
d B A\left(T_{i}, T_{n}\right)= & \prod_{j=i^{*}+1}^{n^{*}}\left(1+\delta^{j} L_{j}^{\delta}\right) \\
= & B A\left(T_{i}, T_{n}\right)\left[\sum_{j=i^{*}+1}^{n^{*}}-\frac{\delta^{j}}{1+\delta^{j} L_{j}} d L_{j}\right. \\
& \left.+\sum_{i+1 \leq j<k \leq n} \frac{\sigma^{j} \delta^{j} L_{j}}{1+\delta^{j} L_{j}} \frac{\sigma^{k} \delta^{k} L_{j}}{1+\delta^{k} L_{k}} \rho_{j, k}^{r} d t\right] \\
= & B A\left(T_{i}, T_{n}\right)\left[\sum_{j=i^{*}+1}^{n^{*}} \frac{\delta^{j}}{1+\delta^{j} L_{j}} L_{j} \sigma^{j}\left(d W^{r, j}-\sum_{k=j+1}^{n^{*}} \frac{\delta^{k} \sigma^{k} L_{k}}{1+\delta^{k} L_{k}} \rho_{j, k}^{r} d t\right)\right] \\
+ & B A\left(T_{i}, T_{n}\right)\left[+\sum_{i^{*}+1 \leq j<k \leq n^{*}} \frac{\sigma^{j} \delta^{j} L_{j}}{1+\delta^{j} L_{j}} \frac{\sigma^{k} \delta^{k} L_{j}}{1+\delta^{k} L_{k}} \rho_{j, k}^{r} d t\right] \\
= & B A\left(T_{i}, T_{n}\right)\left[\sum_{j=i^{*}+1}^{n^{*}} \frac{\delta^{j}}{1+\delta^{j} L_{j}} L_{j} \sigma^{j} d W^{r, j}\right]
\end{aligned}
$$

\section{Correlation}

In order to correlate our multi factor framework, we propose correlation matrix parametrisation:

$$
\begin{aligned}
& d<W_{i}^{F}, W_{j}^{F}>=d t\left(n_{i} n_{j} \sum_{h, l=1}^{n} c_{i, h} c_{j, l} \rho_{h, l}^{Z}+\bar{\rho}_{i, j}^{F}\right) \\
& d<Z_{i}, Z_{j}>=\rho_{i, j}^{Z} d t \\
& d<W_{i}^{F}, Z_{j}>=d t\left(n_{i} \sum_{l=1}^{n} c_{i, l} \rho_{j, l}^{Z}\right) \\
& n_{k}:=\sqrt{1+\sum_{l, h=1}^{n^{*}} c_{k, l} c_{k, h} \rho_{l, h}^{Z}}
\end{aligned}
$$

- $\quad$ Model 0:

$$
\begin{aligned}
& \rho_{i, j}^{Z}=\rho_{\infty}\left(1-\rho_{\infty}\right) e^{-\lambda|i-j|} \\
& \bar{\rho}^{F}=\rho_{\infty}^{F}\left(1-\rho_{\infty}^{F}\right) e^{-\lambda^{F}|i-j|}
\end{aligned}
$$

- $\quad$ Model 1:

$$
\begin{aligned}
\rho_{i, j}^{Z} & =\rho_{\infty}+e^{-\lambda|i-j|} \\
\bar{\rho}^{F} & =\rho_{\infty}^{F}+e^{-\lambda^{F}|i-j|}
\end{aligned}
$$

- Model 2:

$$
\begin{aligned}
& \rho_{i, j}^{Z}=\rho_{\infty} e^{-\lambda|i-j|} \\
& \bar{\rho}^{F}=\rho_{\infty}^{F} e^{-\lambda^{F}|i-j|}
\end{aligned}
$$




\section{Results Monte Carlo benchmarking}

We compare a Monte Carlo routine (path 3,000, steps 300 and 95\% for the confidence interval) to our proxy:

\begin{tabular}{lcc}
\hline $\begin{array}{l}\text { Product } \\
\text { (tenor } \times \text { maturity, strike) }\end{array}$ & $\begin{array}{c}\text { Monte Carlo } \\
\text { (price, upper, lower) }\end{array}$ & $\begin{array}{c}\text { LMM-SABR } \\
\text { (first proxy, second proxy) }\end{array}$ \\
\hline$(3 \times 3,0.9 \%)$ & $(21.173 \%, 21.684 \%, 20.662 \%)$ & $(22.833 \%, 22.854 \%)$ \\
$(1 \times 5,0.9 \%)$ & $(43.052 \%, 43.798 \%, 42.307 \%)$ & $(42.733 \%, 42.743 \%)$ \\
$(4 \times 2,0.9 \%)$ & $(13.761 \%, 14.150 \%, 13.761 \%)$ & $(13.464 \%, 13.481 \%)$ \\
\hline
\end{tabular}

Note: We consider an identity matrix for our testing (so there is no convexity adjustment). 